\title{
Interleukin-29: Just an extra string in the bow of Th17 cells or a target for therapeutic exploitation?
}

\author{
Andrew Johnston ${ }^{1}$
}

Published online: 30 March 2016

(C) Springer-Verlag Berlin Heidelberg 2016

In this issue, Witte and colleagues describe the ability of IL-29, a type III interferon, to induce keratinocyte expression of CXCL9, CXCL10 and CXCL11, three chemokines implicated in the trafficking of activated Th1 T cells into sites of inflammation [1]. Given that Th17 cells may be the principal source of IL-29, this finding both strengthens the role of Th17 cells in antiviral defenses and may help explain the mixed Th1/Th17 phenotype seen in psoriasis, a common inflammatory skin disease.

Immune competence at the epithelial barriers of the gut, lungs and skin takes a concerted effort from the epithelial cells themselves, antibodies, complement, and both resident and infiltrating immune cells to be able to mount a timely response to a barrier breach and invasion by potentially pathogenic microbes. T cells fulfill a vital defensive role in the epithelia, with non-recirculating tissue resident memory $\mathrm{T}$ cells providing rapid specific immune protection against the pathogens most commonly encountered in barrier tissues, supported by innate immune cells and circulating $\mathrm{T}$ cells that can be called upon as needed [2,3]. One trade-off of having a battalion of heavily armed $\mathrm{T}$ cells within the tissues is that loss of control or dysregulation of these cells can initiate and sustain chronic autoimmune and inflammatory diseases, such as Crohn's disease, multiple sclerosis and psoriasis. Over the last decade, the contribution of Th17 cells has been recognized as a vital aspect of immunity at epithelial barriers [4]; however, Th17 cells and IL-17A have also been implicated in a number of their pathologies [5].

Andrew Johnston

andjoh@med.umich.edu

1 Department of Dermatology, University of Michigan, 6427 Medical Science Building I, 1301 Catherine Street, Ann Arbor, MI 48109, USA
IL-17A is a potent inducer of antimicrobial protein and peptide expression by epithelia. In the skin, IL-17A works synergistically with several cytokines including IFN- $\gamma$ [6] and TNF- $\alpha$ [7] to potently drive the induction of a range of antimicrobial peptides and proteins including S100A7, A8 and A9, the cathelicidin LL-37 and human $\beta$-defensins (HBD)-2, 3 and 4. Working in concert, these antimicrobial peptides provide a broad spectrum of antibacterial activity and have also been demonstrated to have efficacy against some viruses [8]. In addition to their antimicrobial activities, the defensins have roles in the regulation of both innate and acquired immunity. HBD-2, 3 and 4 induce pro-inflammatory cytokine expression (IL-6, CXCL10, CCL2, CCL5 and CCL20), and the migration and proliferation of keratinocytes, promoting wound healing [9]. HBD-2 is also a chemoattractant for macrophages, mast cells, activated neutrophils, memory $\mathrm{T}$ cells and dendritic cells (DCs) [10]. The converse is also true: when used at a high enough concentration, chemokines like CCL20 have been shown to possess antimicrobial activities [11].

Plaque psoriasis is a common inflammatory disease of the skin, thought to be driven by Th1 and Th17 T cells unleashing a "cytokine storm", inducing keratinocyte hyperproliferation and perturbed epidermal maturation, resulting in a compromised epidermal barrier characteristic of the disease [12]. Elevated expression of antibacterial peptides and proteins in lesional psoriasis skin is well established, with an extensive inflammatory cytokine milieu driving antimicrobial peptide expression [13], some of which are amongst the most highly upregulated molecules in lesional psoriatic skin [14]. IL-17A is also a potent inducer of an array of antiviral genes such as myxovirus resistance A (MX1) and oligoadenylate synthetases (OAS); thus, Th17 T cells have the potential to contribute to antiviral defenses at epithelial barriers. In addition to increased expression of antimicrobial peptides, antiviral 
protein expression has been shown to be elevated in lesional psoriasis epidermis [15]. Compared with non-lesional skin, psoriasis lesions show elevated MX1, BST2, ISG15 and OAS2 mRNA and protein expression, and although these genes can be induced by a number of cytokines (particularly type I interferons), the antiviral gene expression was found to most closely correlate with the expression of IL-29 [15]. The finding that Th17 cells also secrete IL-29 [15] suggests that Th17 cells have an additional means to bolster antibacterial and antiviral epithelial defenses.

IL-29 is a member of the IL-10 cytokine superfamily, which is an enigmatic collection of structurally related cytokines encompassing IL-10, the IL-20 subfamily (IL-19, IL-20, IL22 , IL-24, IL-26) and the type I interferons (13 IFN- $\alpha$ proteins, one each of IFN- $\beta$, IFN- $\varepsilon$, IFN-K and IFN- $\omega$ ), the type II IFN- $\gamma$ and three type III IFNs- $\lambda$. This family is brought together largely on the basis of their related secondary structure with 6 or $7 \alpha$-helices in an antiparallel conformation. Despite their classification in the same family, these cytokines have little functional overlap, but can be assigned into functional subgroups. This family's figurehead, IL-10, is functionally unique; it is one of the principal immunosuppressive cytokines, preferentially produced by and acting on immune cells. Next, IL-19, IL-20 and IL-24 appear to be pro-inflammatory, produced mostly by monocytes and tissue cells, and acting on tissue cells but not cells of the immune system. Finally, IL-22 and IL-26 appear to be produced only by $\mathrm{T}$ and NK cells and were thought to act only on tissue cells but not immunocytes, but recent work has extended this, finding that IL-26 can bind DNA from dying cells and stimulate plasmacytoid DCs via TLR9 to promote bacterial sensing [16].

The antiviral cytokine IL-29 is one of the trio of type III interferons: IL-29 (IFN- $\lambda 1$ ), IL-28A (IFN- $\lambda 2$ ) and IL-28B (IFN- $\lambda 3)$. All three cytokines signal through a dimeric receptor composed of the IL-10R $\beta$ subunit (also used by IL-10, IL22 and IL-26) and their own dedicated IL-28R $\alpha$ subunit in a manner similar to the type I IFNs, utilizing Jak1 and Tyk2 as adapters initiating phosphorylation and activation of STAT1 and STAT2 which, when active, bind IRF9 forming the IFNstimulated gene factor-3 (ISGF3) transcription factor. The ISGF3 and STAT1 homodimers can bind ISRE and GAS DNA sequences driving the transcription of genes typically induced by type I IFNs (e.g., MX1, OAS2). As such, much of the early investigations into the IFN- $\lambda$ s focused on their type I IFN-like antiviral properties, yet receptor engagement by IL29 also induces the phosphorylation of STAT3, STAT4 and STAT5, suggesting a role not limited to typical antiviral functions. The usage of STAT molecules that are known to be important in the induction of T cell cytokine bias leads investigators to examine the ability of IL-29 to alter the cytokine profile of cultured T cells and DC. They found that IL-29 promotes IFN- $\gamma$ but suppresses IL-13 production from T cells, and promotes IL-12 but suppresses IL-10 expression by DC, thus in principle, having the ability to alter the cytokine bias of T cell responses [17], which could be a means of directing the acquired immune system during viral infections. As mentioned above, IL-29 shows increased expression in psoriasis lesions [15], the source of which appears to be primarily Th17 $\mathrm{T}$ cells although Th1 T cells may also contribute [15]. It has been reported that keratinocytes themselves may also produce IL-29 when challenged with virus [18]. Other documented sources of IL-29 include monocytes, myeloid DC, but not macrophages [19].

In the present work, the authors find that IL-29 preferentially induces keratinocyte expression of CXCL11 [1], and this adds possible new roles for Th17 cells and IL-29 in controlling the immune response in epithelia. Directional leukocyte migration is required for physiological circulation and homing of leukocytes to the lymphoid organs and epithelia. Leukocyte migration is directed by a combination of cellsurface-expressed molecules, termed addressins, such as intercellular adhesion molecules, selectins, integrins and their ligands, and a group of about 50 small and soluble chemotactic proteins, the chemokines and their receptors. CXCR3, or more formally CXCR3A, to distinguish it from the CXCR3B spliced variant that also binds CXCL4 [20], is a $\mathrm{G}_{\alpha \mathrm{i}}$ coupled 7 transmembrane domain receptor for the chemokines CXCL9, 10 and 11. These three ligands function as potent chemoattractants for cells expressing CXCR3A, including Th1 and Th1/Th17 CD4 ${ }^{+} \mathrm{T}$ cells, $\mathrm{CD} 8^{+} \mathrm{T}$ cells and NK cells.

Thus, we can envisage a scenario where Th17 cells infiltrating the skin release IL-29 and induce keratinocyte expression of CXCL11, a potent chemokine for CXCR3 $\mathrm{A}^{+} \mathrm{Th} 1 \mathrm{~T}$ cells, thus promoting further Th1 cell accumulation and helps to account for the mixed Th1/Th17 phenotype reported in psoriasis lesions [6]. Indeed, the current work shows that injection of the murine counterpart of IL-29 (mouse IL-28A) can induce ear swelling and $\mathrm{T}$ cell infiltration of previously sensitized skin, and this is increased when mouse IL-28A is combined with IFN- $\gamma$ [1].

It was recently proposed that chemokines can not only function to attract leukocytes but also influence and possibly re-programme $\mathrm{T}$ cell inflammatory responses [21]. This scenario can be played out at the CXCR3 receptor. While CXCL9, 10 and 11 all utilize CXCR3 on T cells, CXCL11 has the strongest affinity and on binding CXCR3 signals via STAT3/STAT6, using the GATA3 transcription factor resulting in a Treg (IL-10) or Th2 (IL-4) T cell phenotype; CXCL9 and CXCL10 on the other hand drive a Th1/Th17 T cell (IL-17A/IFN- $\gamma$ ) bias. These observations suggest that rather than drawing memory-effector Th1/Th17 cells into tissue to exacerbate inflammation, CXCL11 has the potential to draw in $\mathrm{T}$ cells and by altering their cytokine bias, restrain inflammation. Thus, the production of IL-29 by Th17 cells could be an attempt to engage a regulatory mechanism which alters the cytokine production of infiltrating cells so limiting inflammation (Fig. 1). This alternative scenario is supported 


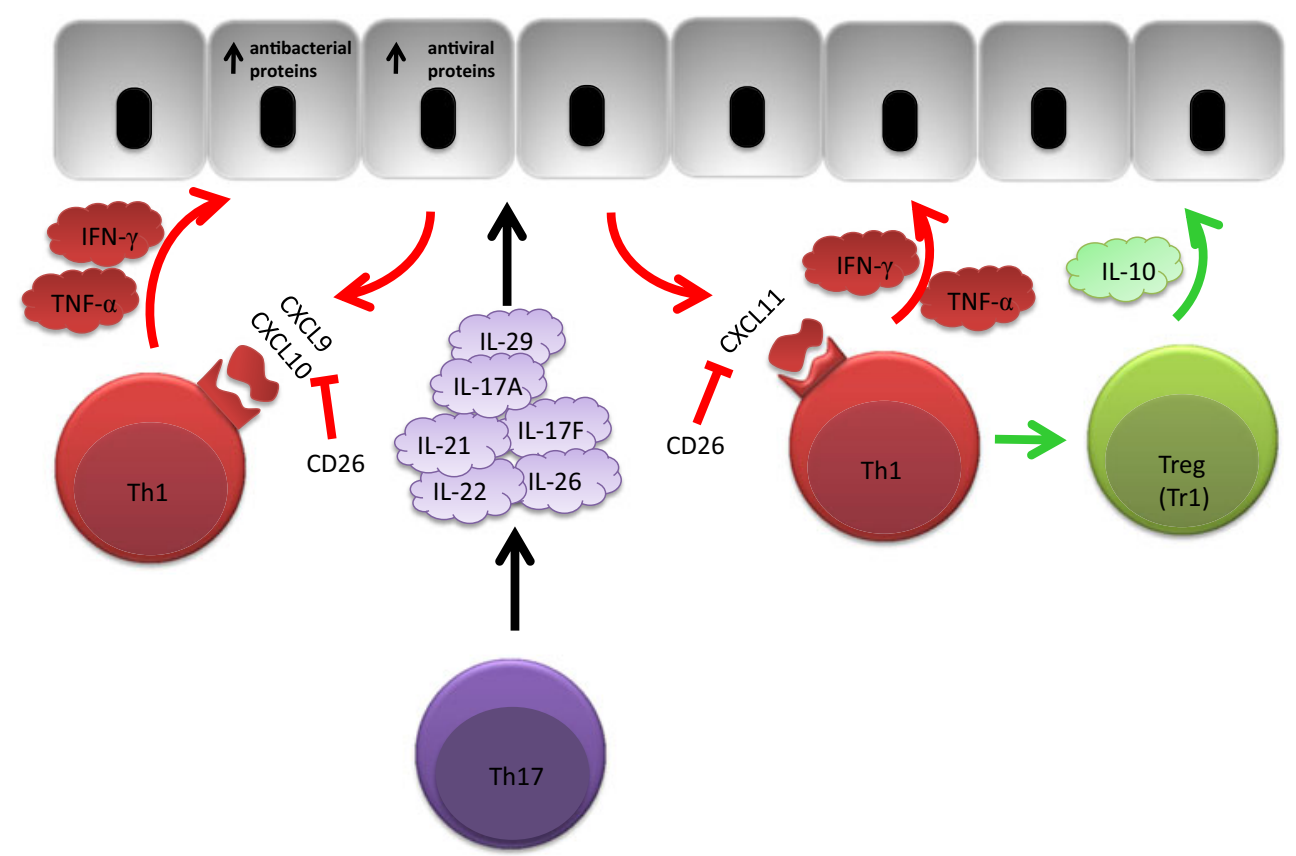

Fig. 1 IL-29 promotes antiviral immunity and via CXCR3A ligands draws in activated Th1 T cells. Th17 cells secrete IL-17A, IL-17F, IL21, IL-22, IL-26 and the type III interferon, IL-29. IL-29 drives antiviral peptide production by epithelial cells (keratinocytes) enhancing antiviral protection and also induces the secretion of CXCL10 and CXCL11 which are potent chemoattractants for cells expressing CXCR3A: Th1 and CD8 T cells, NK cells and Th1/Th17 cells. While CXCL9 and CXCL10

by the observation that TR1 cells also secrete IL-29 [18]; thus, IL-29 may be part of a feedback inhibition pathway that could be exploited to interrupt and reverse the inflammatory cycle.

Although often overlooked, chemokines are also regulated post-translationally by glycosylation and proteolytic processing [22], and the CXCR3A ligands are no exception to this, with the mature chemokines being targets of both CD13 (aminopeptidase N) and CD26 (dipeptidyl peptidase IV). Such processing adds an extra level of regulation to CXCR3A ligand activity: stimulated T cells, while upregulating CXCR3A on their surface, also upregulate CD26 expression, which has been shown to cleave the N termini of CXCL9, 10 and 11 (with the fastest rate for CXCL11) causing a loss of stimulation via CXCR3A, a reduced chemotactic potential but retention of the ability to desensitize the receptor [23].

If the IL-29-CXCL11 axis is indeed anti-inflammatory, there are a number of resources available to exploit this phenomenon: a stabilized CXCL11-Ig fusion protein [21], PEGylated-IL-29 or small molecule inhibition of CD26 (sitagliptin) to potentiate CXCL11 activity [24] could all be employed to re-programme resident or infiltrating Th1/Th17 $\mathrm{T}$ cells in psoriasis lesions towards an anti-inflammatory IL10 -producing phenotype. However, it is currently unclear whether IL-29 and CXCL11 are aiding and abetting a proor anti-inflammatory response, although CXCL11 is increased in the serum of psoriasis patients [1], but it is unknown enhance inflammatory cytokine production from Th1 cells, CXCL11 may have a role in converting Th1 cells to an immunosuppressive IL10-secreting phenotype. An addition layer of control is exerted in this schema by ligand scavenging by the atypical chemokine receptor ACKR3 and rapid inactivation of the ligands by aminopeptidase CD26 (DPP-IV)

whether this is active CXCL11 or proteolytically truncated, inactive chemokine.

Acknowledgments AJ is supported by the National Institute of Arthritis and Musculoskeletal and Skin Diseases of the National Institutes of Health grants AR064765, AR062546 and AR065183, the National Psoriasis Foundation USA and the Babcock Memorial Trust.

\section{References}

1. Witte E, Kokolakis G, Witte K, Warszawska K, Friedrich M, Christou D, Kirsch S, Sterry W, Volk HD, Sabat R et al (2015) Interleukin-29 induces epithelial production of CXCR3A ligands and T-cell infiltration. J Mol Med (Berl). doi:10.1007/s00109-0151367-y

2. Bromley SK, Yan S, Tomura M, Kanagawa O, Luster AD (2013) Recirculating memory $\mathrm{T}$ cells are a unique subset of CD4+ T cells with a distinct phenotype and migratory pattern. J Immunol 190: 970-976

3. Clark RA (2015) Resident memory T cells in human health and disease. Sci Transl Med 7:269rv261

4. Steinman L (2007) A brief history of T(H)17, the first major revision in the $\mathrm{T}(\mathrm{H}) 1 / \mathrm{T}(\mathrm{H}) 2$ hypothesis of T cell-mediated tissue damage. Nat Med 13:139-145

5. Wilke CM, Bishop K, Fox D, Zou W (2011) Deciphering the role of Th17 cells in human disease. Trends Immunol 32:603-611

6. Kryczek I, Bruce A, Gudjonsson J, Johnston A, Aphale A, Vatan L, Szeliga W, Wang Y, Liu Y, Welling T et al (2008) Induction of IL- 
17+ T cell trafficking and development by IFN-gamma: mechanism and pathological relevance in psoriasis. J Immunol 181:4733-4741

7. Chiricozzi A, Guttman-Yassky E, Suarez-Farinas M, Nograles KE, Tian S, Cardinale I, Chimenti S, Krueger JG (2011) Integrative responses to IL-17 and TNF-alpha in human keratinocytes account for key inflammatory pathogenic circuits in psoriasis. J Invest Dermatol 131:677-687

8. Morizane S, Gallo RL (2012) Antimicrobial peptides in the pathogenesis of psoriasis. J Dermatol 39:225-230

9. Niyonsaba F, Ushio H, Nakano N, Ng W, Sayama K, Hashimoto K, Nagaoka I, Okumura K, Ogawa H (2007) Antimicrobial peptides human beta-defensins stimulate epidermal keratinocyte migration, proliferation and production of proinflammatory cytokines and chemokines. J Invest Dermatol 127:594-604

10. Yang D, Chertov O, Bykovskaia SN, Chen Q, Buffo MJ, Shogan J, Anderson M, Schroder JM, Wang JM, Howard OM et al (1999) Beta-defensins: linking innate and adaptive immunity through dendritic and T cell CCR6. Science 286:525-528

11. Yang D, Chen Q, Hoover DM, Staley P, Tucker KD, Lubkowski J, Oppenheim JJ (2003) Many chemokines including CCL20/MIP3alpha display antimicrobial activity. J Leukoc Biol 74:448-455

12. Elder JT, Bruce AT, Gudjonsson JE, Johnston A, Stuart PE, Tejasvi T, Voorhees JJ, Abecasis GR, Nair RP (2010) Molecular dissection of psoriasis: integrating genetics and biology. J Invest Dermatol 130:1213-1226

13. Baliwag J, Barnes DH, Johnston A (2015) Cytokines in psoriasis. Cytokine 73:342-350

14. Mee JB, Johnson CM, Morar N, Burslem F, Groves RW (2007) The psoriatic transcriptome closely resembles that induced by interleukin-1 in cultured keratinocytes: dominance of innate immune responses in psoriasis. Am J Pathol 171:32-42

15. Wolk K, Witte K, Witte E, Raftery M, Kokolakis G, Philipp S, Schonrich G, Warszawska K, Kirsch S, Prosch S et al (2013) IL29 is produced by $\mathrm{T}(\mathrm{H}) 17$ cells and mediates the cutaneous antiviral competence in psoriasis. Sci Transl Med 5:204ra129
16. Meller S, Di Domizio J, Voo KS, Friedrich HC, Chamilos G, Ganguly D, Conrad C, Gregorio J, Le Roy D, Roger T et al (2015) $\mathrm{T}(\mathrm{H}) 17$ cells promote microbial killing and innate immune sensing of DNA via interleukin 26. Nat Immunol 16:970-979

17. Jordan WJ, Eskdale J, Srinivas S, Pekarek V, Kelner D, Rodia M, Gallagher G (2007) Human interferon lambda-1 (IFN-lambda1/IL29) modulates the Th1/Th2 response. Genes Immun 8:254-261

18. Wolk K, Witte K, Sabat R (2010) Interleukin-28 and interleukin-29: novel regulators of skin biology. J Interferon Cytokine Res 30:617-628

19. Wolk K, Witte K, Witte E, Proesch S, Schulze-Tanzil G, Nasilowska K, Thilo J, Asadullah K, Sterry W, Volk HD et al (2008) Maturing dendritic cells are an important source of IL-29 and IL-20 that may cooperatively increase the innate immunity of keratinocytes. J Leukoc Biol 83:1181-1193

20. Lasagni L, Francalanci M, Annunziato F, Lazzeri E, Giannini S, Cosmi L, Sagrinati C, Mazzinghi B, Orlando C, Maggi E et al (2003) An alternatively spliced variant of CXCR3 mediates the inhibition of endothelial cell growth induced by IP-10, Mig, and I-TAC, and acts as functional receptor for platelet factor 4. J Exp Med 197:1537-1549

21. Zohar Y, Wildbaum G, Novak R, Salzman AL, Thelen M, Alon R, Barsheshet Y, Karp CL, Karin N (2014) CXCL11-dependent induction of FOXP3-negative regulatory $\mathrm{T}$ cells suppresses autoimmune encephalomyelitis. J Clin Invest 124:2009-2022

22. Mortier A, Van Damme J, Proost P (2008) Regulation of chemokine activity by posttranslational modification. Pharmacol Ther 120: 197-217

23. Ludwig A, Schiemann F, Mentlein R, Lindner B, Brandt E (2002) Dipeptidyl peptidase IV (CD26) on T cells cleaves the CXC chemokine CXCL11 (I-TAC) and abolishes the stimulating but not the desensitizing potential of the chemokine. J Leukoc Biol 72:183191

24. Mortier A, Gouwy M, Van Damme J, Proost P, Struyf S (2016) CD26/dipeptidylpeptidase IV-chemokine interactions: doubleedged regulation of inflammation and tumor biology. J Leukoc Biol. doi:10.1189/jlb.3MR0915-401R 